\title{
Research and Design of Software Package "Medved.Telemed"
}

\author{
Konstantin A. Aksyonov ${ }^{1, \text { a) }}$, Julia V. Yudina ${ }^{1, \text { b) }}$, Konstantin Y. Suslov ${ }^{1, \text { c), }}$ \\ Olga P. Aksyonova ${ }^{1, d}$, Polina E. Ziomkovskaia ${ }^{1, \text { e) }}$ \\ ${ }^{1}$ Yeltsin Ural Federal University, Yekaterinburg, Russia \\ a)Corresponding author: k.a.aksyonov@urfu.ru \\ b)juliashka95@list.ru \\ c)wiper99@mail.ru \\ d)bpsim.dss@gmail.com \\ e)polina.ziomk@yandex.ru
}

\begin{abstract}
The work is devoted to design of the software package "Medved.Telemed". In this paper, the main types of architectures and their application situations were studied. Also, we study the main competitors, the legal basis. And current trends in the development of healthcare in the field of digital medicine. Based on the knowledge achieved and identified requirements, a hybrid architecture of a new information system has been developed. The system which is a combination of several architectural patterns, each of which at its own level provides the flexibility and scalability of each component and solution in the whole.
\end{abstract}

\section{INTRODUCTION}

Development of the application architecture is one of key steps in creating a new application. Wrong description of the system requirements, key scenarios, and misconception about the long-term perspectives may lead to impossibility of the architecture application. Moreover, its application could not be developed in accordance with new conditions, or making changes in it will take a long time since its mortgaged drawbacks. As a result, people can lose their place in the market giving a way to the competitors.

What should be a "good" architecture? It should simplify the process of developing and maintaining the system. Good architecture makes it easy to extend, modify, and update the application.

Developed by the group of companies HOST, the Russian platform Medved automates the work of regional health care authorities and the provision of electronic services to the public. This system has such functions:

- Centralized collection and storage of health indicators in the region.

- Detailing the summary data to a specific medical case.

- Automation of preparation processes of the analytical subdivisions.

- Data visualization using the geographic information systems.

- Analytical and forecasting tools for the operational and high-quality management decisions.

- Instrumental and informational support of theexpert and analytical activities of management and specialists.

The main function of the program "Medved.Telemed" is in providing the users with a possibility of recording the medical services and conducting the telemedicine consultations with a doctor. Initially, the application allowed only telemedicine consultations, at this stage of a Minimum viable product (MVP) approach, the first testing of the application was conductedby users. The test of results showed interest from the customer and users to the system, that made a good start of its development. However, it immediately became clear that the current application would 
not be able to withstand competition in the rapidly growing medical software market, since the architecture did not cover the identified requirements with the MVP approach.

The relevance of the design of this solution is due to the increasing demand for telemedicine among both the public and private medical organizations. The Telemedicine Act, which entered into force in 2018, initiated the development of the telemedicine systems for the public health sector and strengthened the position and demand for these systems in the private sector.

\section{REQUIREMENT SELICITATION}

The study of analogues revealed the following main roles in the solution:

- patient;

- doctor;

- collaborator ofthe insurancecompany;

- operator ofthemunicipalunit.

In addition to the abovementioned roles in the system, it is also needed to adda role of the administrator who can manage and configure the system. Since there can be several municipal units (MUs) per one instance, it makes sense to single out the roles

- administrator of the municipal unit-responsible for setting hospital parameters;

- administrator of the instance- responsible for the operation of all hospitals' instance.

Functions of each role are unique, so, each of them must have its own user interface optimized for their needs. Administrators can be combined in a single interface, since the role of "Administrator of the instance" is just an extension of the role of the "Administrator of the municipal unit (MU)".

Business requirements for the developed application derive primarily from a legislative framework of telemedicine and basics of health [1]. Based on requirements for the organization of telemedicine in order $965 \mathrm{n}$, the system should provide:

- authorization of participants in telemedicine consultations through a unified system of identification and authentication (USIA);

- $\quad$ signing of documents that are being sent by doctors with a qualified enhanced electronic signature;

- storage of documents forwarded in the course of five years;

- patients informing about the medical organization providing telemedicine services (name, address, telephone, email) [2];

- inform patients about physicians providing telemedicine services (information about education, place of work, work experience);

- storage of all content generated in the process of using the system at regional facilities.

In addition to requirements associated with the telemedicine order, there are also requirements dictated by the market:

- it should be possible to quickly add new services for patient;

- it should be possible for a patient who has entered the application through the SaaS-instance to receive all his or her data from the regional instance;

- it should be possible to connect different authorizations, since the customer may already have its own integration through the USIA. 


\section{DECISION DESCRIPTION}

Roles, which are outlined in the previous section have different capabilities, respectively, and user interfaces for these roles will be different. Based on the service-oriented architecture of the application, it is necessary to form the composition of modules.

A doctor and a patient have different interfaces, so it would be possible to spread them into different modules. However, since they have to communicate in chat and via video link, it was decided to combine a doctor and a patient into one module (after referred to as Core) making for each one a separate interface [3]. Transmission of messages within a single module simplifies the implementation, otherwise, it would be necessary to use integration to send messages. In addition, doctors and patients have a single content: the chat rooms, medical card; so, we avoid duplication of the data.

An operator and administrators must also have their own separate modules. Despite the fact that functions of the operator and the administrator are similar, it is worthwhile to locate them into separate modules, since not every region needs the operator MU module. Therefore, it should be possible to easily disable its functions. The easiest option is to bring it to a separate service [4, 7], an alternative solution is, for example, the cloud service [6].

Functions of an insurance company employee are unique, so, it is also put into a separate module.

In addition to modules with the users' interfaces, there is an authorization module and a video conferencing one (after referred to as $\mathrm{CM}$ ). Thus, the platform consists of the following modules:

- $\quad$ system core;

- administration of module;

- insurance module;

- CM module.

It was decided to separate referenced data into a separate database

- list of clinics;

- list of doctors;

- list of doctors' specializations;

- list of available services;

- methods for services.

These data are relatively static. Therefore, in order not to request them in external sources each time, they are stored in the separate database, and their modification is carried out through the administrator module.

In addition to the custom modules, the system also has the following set of services:

- $\quad$ authorization service;

- doctor/not doctor check-up service;

- voluntary health insurance check service;

- user information service;

- record entry services.

To implement the interaction between the regional instance and the SaaS (software as a service), an instance connector is introduced, it is a kind of a gateway that is responsible for data transfer between instances, as well as for the user authorization and notifications.

Thus, we obtained the overall architecture of the software package "Medved.Telemed", presented in Fig. 1.This approach to the design of an architecture of the telemedical system «Medved.Telemed» was used in the development of an automated system for the production of metallurgical products [8-10]. The overall architecture of the Medved.Telemed telemedicine system consists of the

- moduls;

- data bases;

- servises;

- instance connector. 


\section{CONCLUSION}

In the paper, the hybrid architecture of the «Medved.Telemed» software package was designed on the basis of the requirements and existing architecture patterns based on combining principles of multi-layer organization. Here we used the service-oriented approach andthe event management pattern. In this architecture, there are possibilities for its expansion and modification using frames, which allow quickly connecting new modules to the system, as well as, easily replacing existing interface pages for each client. Further work will be related to implementation of this architecture and entry into the market of the telemedicine applications. The «Medved.Telemed» system is being introduced in Russia by the "HOST" group of companies.

\section{ACKNOWLEDGMENTS}

This work is supported by Act 211 Government of the Russian Federation, contract № 02.A03.21.0006.

\section{REFERENCES}

1. Order of the Ministry of Health of Russia of November 30, 2017 N 965n "On approval of the procedure for organizing and providing medical care using telemedicine technologies." - Access mode: http://www.consultant.ru/document/cons_doc_LAW_287515/.

2. Federal Law "On Amendments to Certain Legislative Acts of the Russian Federation on the Application of Information Technologies in the Field of Health Protection" of 29.07.2017 N 242-FZ (last revised). - Access mode: http://www.consultant.ru/document/cons_doc_LAW_221184/.

3. J.D. Meier et al. "Microsoft Application Architecture Design Guide", 2009.

4. Company blog IT-GRAD, Analysis and design of systems [Electronic resource]. - Access mode: https://habr.com/ru.

5. Software architecture patterns [Electronic resource]. - Access mode: https://www.oreilly.com/ideas/softwarearchitecture-patterns/page/3/event-driven-architecture

6. V.V. Devyatkov, S.A. Vlasov, T.V. Devyatkov, "Cloud Technology in Simulation Studies: GPSS Cloud Project" ,Proceedings of the 7th IFAC Conference on Manufacturing Modeling, Management, and Control, Vol. 7. Part 1, (Saint Petersburg ,Russia,2013), pp. 637-641.

7. A.Kondratyev, K.Aksyonov, N. Buravova, O. Aksyonova, "Cloud-based microservices to decision support" The tenth International Conference on Ubiquitous and Future Networks, (Prague, Czech Republic, 2018), pp. 389-394.

8. A.Borodin, Y.Kiselev, S.Mirvoda, S.Porshnev, "On design of domain-specific query language for the metallurgical industry",Proceedings of the 11th International Conference BDAS: Beyond Databases, Architectures and Structures: Communications in Computer and Information Science, pp. 505-515.

9. A.Borodin, S.Mirvoda, I.Kulikov, S.Porshnev, "Optimization of memory operations in generalized search trees of PostgreSQL",Proceedings of the International Conference: Beyond Databases, Architectures and Structures, pp. 224-232.

10. K.Aksyonov, A.Antonova, "Application of the process parameter analysis tree for technological process analysis",2018 Ural Symposium on Biomedical Engineering, Radioelectronics and Information Technology, (Yekaterinburg, Russia,2018), pp.212-215. 